\title{
ABORDAGEM GERAL TRAUMA ABDOMINAL
}

\author{
MANAGEMENT OF THE ABDOMINAL TRAUMA
}

Gerson Alves Pereira Júnior ${ }^{1}$, Wilson José Lovato², Júlia Batista de Carvalho ${ }^{3}$, Marcos Fúlvio Vieira Horta ${ }^{4}$

\begin{abstract}
'Médico Assistente, Unidade de Emergência (UE), Hospital das Clínicas da Faculdade de Medicina de Ribeirão Preto - USP (HCFMRPUSP). Docente, Emergências Médicas e Habilidades Cirúrgicas, Curso de Medicina da Universidade de Ribeirão Preto (UNAERP). ${ }^{2}$ Médico Assistente, UE - HCFMRP-USP. Docente. Semiologia Geral e do Adulto. Curso de Medicina- UNAERP. ${ }^{3}$ Médica residente de Medicina Intensiva - UNAERP. ${ }^{4}$ Docente, Cirurgia Plástica, Curso de Medicina - UNAERP.

CorRespondência: Dr. Gerson Alves Pereira Júnior. Rua Bernardino de Campos, 1000. Higienópolis. CEP 14030-150 Ribeirão Preto - SP e-mail: gersonapj@gmail.com
\end{abstract}

Pereira Júnior GA, Lovato WJ, Carvalho JB, Horta MFV. Abordagem geral trauma abdominal. Medicina (Ribeirão Preto) 2007; 40 (4): 518-30, out./dez.

RESUMO: O trauma abdominal é freqüentemente encontrado em situações de emergência. A falta de história adequada do mecanismo de trauma e a presença de lesões que podem ter dor irradiada para o abdome ou a alteração do estado mental, devido a trauma cranioencefálico ou intoxicação por drogas depressoras do sistema nervoso central, podem dificultar o diagnóstico e o tratamento do trauma abdominal. Os pacientes que são vítimas de trauma, freqüentemente, têm lesões intra e extra-abdominais associadas.

Este artigo de revisão irá discutir a abordagem geral do atendimento dos pacientes com traumas abdominais contusos e penetrantes, incluindo as opções de testes diagnósticos e as considerações acerca do tratamento inicial.

Descritores: Traumatismos Abdominais. Ferimentos Penetrantes. Ferimentos não Penetrantes. Avaliação. Tratamento.

\section{1- INTRODUÇÃO}

O traumatismo abdominal é responsável por um número expressivo de mortes evitáveis. A cavidade intraperitoneal, juntamente com a cavidade torácica, o espaço retroperitoneal (sobretudo na presença de fraturas de bacia) e as fraturas de ossos longos, são os locais do organismo que comportam sangramentos capazes de levar à morte por choque hemorrágico ${ }^{1}$.

O mecanismo de trauma, a localização da lesão e o estado hemodinâmico do paciente determinam o momento da avaliação do abdome ${ }^{1}$.

Boa parte dos quadros de hemoperitônio decorrentes de uma lesão visceral abdominal são oligossintomáticos ${ }^{1}$. Além disso, os sintomas abdominais relacionados ao traumatismo, muitas vezes, são obscurecidos por lesões associadas com dor referida ou, por alterações do nível de consciência, principalmente, decorrentes do trauma craniano, o que dificulta a sua avaliação. Portanto, uma avaliação rigorosa do abdome e uma correta orientação irão reduzir os erros na interpretação e os impactos desfavoráveis na evolução do paciente ${ }^{1,2}$.

\section{2- HISTÓRIA E MECANISMO DE TRAUMA}

As informações colhidas da vítima, quando possível, ou dos socorristas que efetuaram operação de resgate são valiosas. As noções da biomecânica do trauma, o estado inicial da vítima no local de atendimento, diagnósticos realizados, a resposta à infusão de fluidos no início e o tempo decorrido desde o trauma irão auxiliar na suspeita de lesão abdominal ${ }^{2}$.

Nos casos de colisões automobilísticas, as se- 
guintes informações são fundamentais para se antecipar o padrão de lesões:

1- o tipo de colisão (frontal, lateral, traseira, angular e capotamento);

2- localização e intensidade da deformação externa do veículo;

3- presença de vítimas ejetadas;

4- morte de um dos ocupantes do veículo;

5- uso de dispositivos de segurança veicular (cinto de segurança, air-bag);

6- grau de deformação do espaço interno do veículo ocupado pelas vítimas; e

7- o posicionamento das mesmas dentro do veículo ${ }^{1,2}$.

Nas colisões motociclísticas são importantes as informações sobre o uso e tipo de capacete pela vítima, a superfície onde ocorreu o trauma e outros eventos relacionados ao trauma, como a ejeção e atropelamento subseqüente ${ }^{1,2}$.

No caso de quedas, as informações sobre a altura envolvida, superfície onde ocorreu, possíveis anteparos de trauma antes de atingir o solo e a parte do corpo que primeiramente sofreu o impacto são fundamentais ${ }^{1,2}$.

Nos ferimentos penetrantes por arma branca, o sexo do agressor, número de lesões, o lado e posição do corpo atingido e o tipo de arma (tamanho e diâmetro) são de extrema valia na avaliação inicial. Nos ferimentos penetrantes por arma de fogo, o tipo de arma, calibre, distância de disparo, número de lesões, locais do corpo atingidos e o exame dos orifícios de entrada e/ou saída dos projéteis auxiliam na individualização das decisões ${ }^{1,2}$.

\section{1- Trauma contuso}

No trauma contuso de abdome, as vísceras são submetidas a movimentos de aceleração, desaceleração, compressão e cisalhamento nas diversas direções. As vísceras parenquimatosas, tanto pelo seu tamanho, como pelo peso são, particularmente, susceptíveis às lacerações, cisalhamentos de pedículos vasculares, esmagamentos e roturas no local de transição e fixação anatômica. O baço é o órgão lesado em cerca de 40 a $55 \%$ das laparotomias por trauma contuso e o fígado em 35 a 45\%. Menos freqüentemente, as vísceras ocas podem ser lesadas no trauma contuso $^{1,2}$.

\section{2- Trauma penetrante}

Os agentes penetrantes propiciam lesões de forma direta, em função de sua trajetória e das estruturas que atravessam. A trajetória é limitada aos ór- gãos anatomicamente adjacentes à lesão nos ferimentos por arma branca, enquanto que os ferimentos por projéteis de arma de fogo podem apresentar trajetórias diversas, além de provocarem lesões teciduais pela força de cavitação ${ }^{1,2}$.

Os ferimentos por arma branca acometem mais freqüentemente o fígado (40\%), intestino delgado $(30 \%)$, diafragma (20\%) e cólon $(15 \%)$. Os ferimentos por arma de fogo causam mais danos intra-abdominais devido à extensão da sua trajetória e a maior energia cinética dissipada, tendo como principais sedes de lesão, o intestino delgado (50\%), cólon (40\%), fígado $(30 \%)$ e estruturas vasculares abdominais $(25 \%)^{3,4}$.

Os ferimentos de dorso, períneo, nádegas e tórax podem comprometer estruturas abdominais. Assim, todos os ferimentos que comprometem essas regiões devem ser considerados abdominais, até prova em contrário ${ }^{3,4}$.

Os ferimentos do tórax inferior podem acometer a região de transição tóraco-abdominal, que pode ser limitada superiormente por uma linha que passa pelo $4^{\circ}$ espaço intercostal, anteriormente (linha intermamilar), e pelo $7^{\circ}$ espaço intercostal, posteriormente (ponta das escápulas) e seu limite inferior é dado pelo rebordo costal. Nessa região, o diafragma executa seus movimentos, o que explica o possível comprometimento torácico e abdominal dos ferimentos desse segmento do tronco ${ }^{1}$.

A possibilidade de lesão varia de acordo com o tipo de agente penetrante. Nos ferimentos por arma branca da parede anterior do abdome, a incidência de lesão é de 30 a $40 \%$. Nos ferimentos do flanco e da região lombar, o percentual de lesões é ainda menor, atingindo 18 a 23\%. Assim, nos ferimentos abdominais penetrantes por arma branca, justifica-se a adoção de conduta seletiva para evitar laparotomias desnecessárias ${ }^{2}$.

Os ferimentos abdominais por arma de fogo comportam uma taxa de lesão interna de até $97 \%$, sendo o poder destruitivo maior nos ferimentos por armas militares do que por armas civis. Deste modo, salvo em raras exceções, a laparotomia exploradora é mandatória neste tipo de mecanismo de trauma, para o controle de sangramentos e contaminação intestinal ${ }^{4}$.

\section{3- SINAIS E SINTOMAS}

Todo paciente traumatizado deve ser atendido seguindo-se a sistematização do exame primário do Advanced Trauma Life Support (ATLS) ${ }^{1}$. 
$\mathrm{Na}$ avaliação do paciente com suspeita de trauma abdominal, todos os esforços concentram-se em se fazer o diagnóstico da presença de lesão abdominal, sendo de menor importância o diagnóstico topográfico específico da lesão ${ }^{1}$.

O quadro clínico mais freqüente é a presença de choque hemorrágico sem causa aparente ${ }^{1}$. Devemos excluir outras causas de choque hemorrágico no tórax, retroperitônio/bacia e ossos longos. Também deve-se excluir causas de choque não hemorrágico ${ }^{1}$.

$\mathrm{Na}$ investigação do paciente com suspeita de trauma abdominal, os sinais no exame físico podem não ser aparentes na admissão. Cerca de $40 \%$ dos pacientes com hemoperitônio de considerável volume podem não apresentar manifestações clínicas na avaliação inicial ${ }^{4,5}$.

O uso de drogas opióides deve ser evitado em pacientes com hipovolemia, trauma craniencefálico ou trauma abdominal, pois podem agravar a hipotensão, levar a depressão respiratória e impedir a valorização clínica dos achados ${ }^{4,5}$.

O abdome deve ser completamente inspecionado nas suas faces anterior e posterior como também as nádegas e a região perineal. A presença de escoriações, contusões, hematomas localizados e ferimentos abertos são sugestivos de trauma e devem ser bem caracterizados ${ }^{4,5}$.

A ausculta do abdome permite confirmar a presença ou ausência de ruídos hidroaéreos. A presença de sangue ou conteúdo gastrintestinal pode produzir íleo, resultando em diminuição dos ruídos hidroaéreos ${ }^{4,5}$.

A percussão do abdome pode demonstrar som timpânico devido à dilatação gástrica no quadrante superior esquerdo ou macicez difusa quando hemoperitônio está presente ${ }^{4,5}$.

A rigidez abdominal voluntária pode tornar o exame físico abdominal não confiável. De maneira contrária, a rigidez involuntária da musculatura abdominal é um sinal confiável de irritação peritoneal. A dor à descompressão brusca, geralmente, indica uma peritonite estabelecida pelo extravasamento de sangue ou conteúdo gastrintestinal. A presença de um útero gravídico e a determinação da sua altura podem estimar a idade fetal ${ }^{4,5}$.

Os sinais de irritação peritoneal podem indicar a necessidade de cirurgia, porém na presença de estabilidade hemodinâmica, particularmente, em traumas contusos, podemos realizar a tomografia computadorizada de abdome para estadiamento anatômico das lesões com a possibilidade de tratamento não operatório de traumas de vísceras parenquimatosas ${ }^{6}$.

A compressão manual das cristas ilíacas ânterosuperior pode mostrar movimento anormal ou dor óssea que sugere a presença de fratura pélvica em pacientes com trauma contuso do tronco ${ }^{1}$.

O toque retal deve ser parte obrigatória do exame físico do paciente politraumatizado, dando informações como a presença de sangue na luz retal, fragmentos de ossos pélvicos que penetram o reto, a crepitação da parede posterior do reto (retropneumoperitôneo), a atonia esfincteriana (lesão medular) e a posição alta da próstata (lesão uretral). Nos pacientes com ferimento abdominal penetrante por arma branca ou de fogo, a identificação da presença de sangue no toque retal mostra que houve perfuração intestinal, cujo tratamento é cirúrgico, sem a necessidade de outras investigações especifícas ${ }^{1}$.

Da mesma forma, o exame vaginal na mulher pode caracterizar sinais de violência sexual, sangramentos e a presença de espículas ósseas decorrentes de fraturas pélvicas ${ }^{1}$.

O exame do períneo e do pênis pode demonstrar uretrorragia e hematoma de bolsa escrotal, sugerindo fortemente a presença de lesão uretral, o que contra-indica a sondagem vesical ${ }^{1}$.

Os ferimentos penetrantes da região glútea associam-se com uma incidência maior de $50 \%$ de lesão abdominal significante ${ }^{7}$.

O exame físico abdominal é importante, mas não é confiável. É importante ressaltar que o encontro de algum achado positivo no exame físico do abdome deve sugerir a presença de uma lesão interna, porém sua ausência não afasta a possibilidade de lesão. Assim, o seguimento clínico apropriado e a utilização de exames complementares, particularmente, os de imagem, são fundamentais ${ }^{5,6}$.

Em algumas situações clínicas relacionadas, o abdome não pode ser avaliado adequadamente pelo exame físico ou os achados não são confiáveis ${ }^{1,5,6}$.

1- alteração do nível de consciência por traumatismo cranioencefálico, etilismo agudo ou abuso de drogas depressoras do sistema nervoso central;

2- pacientes com lesões da coluna cervical;

3- fratura costal baixa ou pélvica que podem confundir o exame físico abdominal, devido à dor irradiada ou referida; e

4- anestesia para a realização de procedimentos cirúrgicos extra-abdominais. 


\section{4- MEDIDAS AUXILIARES DO EXAME AB-} DOMINAL

\section{1- Sonda nasogástrica}

O emprego da sonda gástrica visa a descomprimir o estômago, diminuindo o risco de aspiração. Pode, entretanto, detectar a presença de sangue levantando a suspeita de lesão no trato digestivo superior, uma vez afastado o sangramento deglutido das fraturas nasomaxilofaciais. As contra-indicações para a instalação da sonda nasogástrica são as fraturas da face média e a suspeita da fratura da base do crânio ${ }^{1}$. Nestes casos, deve-se utilizar a via orogástrica ${ }^{1}$.

\section{2- Sonda vesical}

A sondagem vesical permite o controle do débito urinário, que pode ser utilizado para se avaliar a resposta clínica à reposição volêmica. A cateterização vesical também é importante para se avaliar o aspecto da urina. A presença de hematúria macroscópica indica a possibilidade de lesão do trato urinário alto ou baixo ${ }^{1}$.

O emprego da sonda vesical está contra-indicado quando existem sinais sugestivos de lesão uretral que são: uretrorragia, o hematoma de bolsa escrotal ou de períneo e a próstata em posição elevada no toque retal em pacientes do sexo masculino. Diante desses achados, uma uretrocistografia injetora deve ser realizada antes da tentativa de passagem da sonda vesical. Caso não seja detectada lesão na uretra, podese passar a sonda vesical ${ }^{1}$.

\section{5- DIAGNÓSTICO}

$\mathrm{O}$ avanço na tecnologia dos exames de imagem e a difusão destes exames pelos serviços de atendimento médico de emergência permitiram o diagnóstico mais rápido e preciso das lesões abdominais, diminuindo o risco de lesões desapercebidas ${ }^{8}$.

\section{1- Traumas contusos}

Os pacientes com instabilidade hemodinâmica e sinais óbvios de trauma abdominal devem ser submetidos à exploração cirúrgica imediata1.

Os pacientes conscientes, com mecanismo de trauma pouco sugestivo e sem achados suspeitos de trauma abdominal no exame físico, não necessitam ser investigados. Caso não haja outras suspeitas, podem receber alta hospitalar ou ser mantidos em observação clínica com exames clínicos repetidos. Todas as informações devem ser anotadas no prontuário médico e comparadas com as avaliações anteriores, de preferência, realizadas pelo mesmo médico, pois as alterações observadas no exame físico podem determinar uma investigação diagnóstica específica ou até a indicação cirúrgica ${ }^{1,4}$.

Em todos os pacientes com rebaixamento do nível de consciência, com sinais positivos no exame físico ou com mecanismo de trauma que levem a suspeita de trauma abdominal devem ser submetidos à investigação diagnóstica para a confirmação ou exclusão de lesões abdominais. A investigação diagnóstica a ser realizada vai depender do estado hemodinâmico após a reposição de fluidos e podemos ter duas situações clínicas:

1- nos pacientes que mantém instabilidade hemodinâmica mesmo após agressiva reposição de fluidos, o lavado peritoneal diagnóstico ou o ultra-som de abdome devem ser utilizados, pois são exames muito sensíveis, para detectarem a presença de sangue ${ }^{9}$;

2- nos pacientes que mantém a estabilidade hemodinâmica após a reposição de fluidos, o exame de escolha é a tomografia computadorizada de abdome, pois sua alta especificidade permite o adequado estadiamento anatômico das as lesões abdominais, o que pode possibilitar o tratamento não operatório das lesões de vísceras parenquimatosas ${ }^{8}$.

\section{2- Traumas penetrantes}

Os pacientes com instabilidade hemodinâmica e sinais óbvios de trauma abdominal, tais como a evisceração com exposição de alças intestinais ou epíplon e a peritonite generalizada devem ser submetidos à exploração cirúrgica imediata ${ }^{1}$.

A abordagem diagnóstica e de tratamento do trauma penetrante está lentamente mudando de uma indicação de exploração cirúrgica imediata para uma conduta conservadora em casos selecionados, particularmente, nos ferimentos por arma branca. Esta alteração da conduta tem sido possível graças a uma maior acurácia do diagnóstico clínico, laboratorial e, particularmente, radiológico na avaliação e estadiamento de cada caso ${ }^{10}$.

A utilização do exame físico isoladamente ou junto com diferentes métodos diagnósticos têm permitido a redução das laparotomias não terapêuticas e a eliminação das laparotomias negativas ${ }^{10}$.

\subsection{1- Ferimentos por arma branca da parede an- terior do abdome}

A maior parte destes pacientes apresentam estabilidade hemodinâmica e queixam-se apenas de dor no local do ferimento. Nestes casos, está indicada a 
exploração local do ferimento. É realizada após antissepsia e anestesia local, podendo-se necessitar ou não de ampliação do ferimento com bisturi. Tal procedimento é realizado na sala de trauma e o objetivo é saber se houve ou não a violação da cavidade peritoneal (Figura 1). Em caso de dúvida, o paciente pode ser mantido em observação clínica com exames físicos repetidos, realização do lavado peritoneal diagnóstico ou submetido à laparotomia exploradora ${ }^{3,11}$.

Dependendo do tipo de arma branca, como estiletes, por exemplo, pode ser mais difícil reconhecer o trajeto e a violação peritoneal e, nestes casos, a laparotomia exploradora é a melhor opção ${ }^{11}$.

Os pacientes obesos ou pouco colaborativos podem ser submetidos a anestesia geral para a realização de laparoscopia. Se houver penetração fascial na exploração local do ferimento ou penetração peritoneal na laparoscopia, está indicada a laparotomia exploradora ${ }^{12}$.

Esta conduta seletiva do ferimento abdominal por arma branca tem permitido a redução do número de laparotomias negativas ou não terapêuticas ${ }^{12}$.

\subsection{2- Ferimentos por arma branca dos flancos ou do dorso}

Devido à espessa musculatura nesta região, a exploração digital torna-se ineficiente e os estudos contrastados do trajeto dos ferimentos (trajetografia) mostraram-se desanimadores e inconsistentes. Assim, a melhor opção para os pacientes com suspeita de lesão abdominal nestes casos é a realização de tomografia computadorizada do abdome com triplo contraste (via oral, endovenosa e por enema retal). Este tipo de tomografia computadorizada é um exame trabalhoso e deve ter a completa contrastação do cólon, tendo acurácia comparável ao exame físico seriado, porém permite o diagnóstico mais precoce da lesão ${ }^{13,14}$.

Um achado positivo na tomografia ocorre quando há evidência de violação peritoneal ou lesão de estruturas retroperitoneais como o cólon, vasos calibrosos e o trato urinário ${ }^{14}$.

Os pacientes com achado positivo na tomografia, exceto aqueles com lesões isoladas do fígado ou líquido livre intraperitoneal, devem ser submetidos à laparotomia exploradora ${ }^{14}$.

\subsection{3- Ferimentos por arma de fogo}

A grande maioria dos pacientes com ferimentos por arma de fogo no abdome requer laparotomia imediata para controle do sangramento e da contaminação intestinal ${ }^{4,15}$.

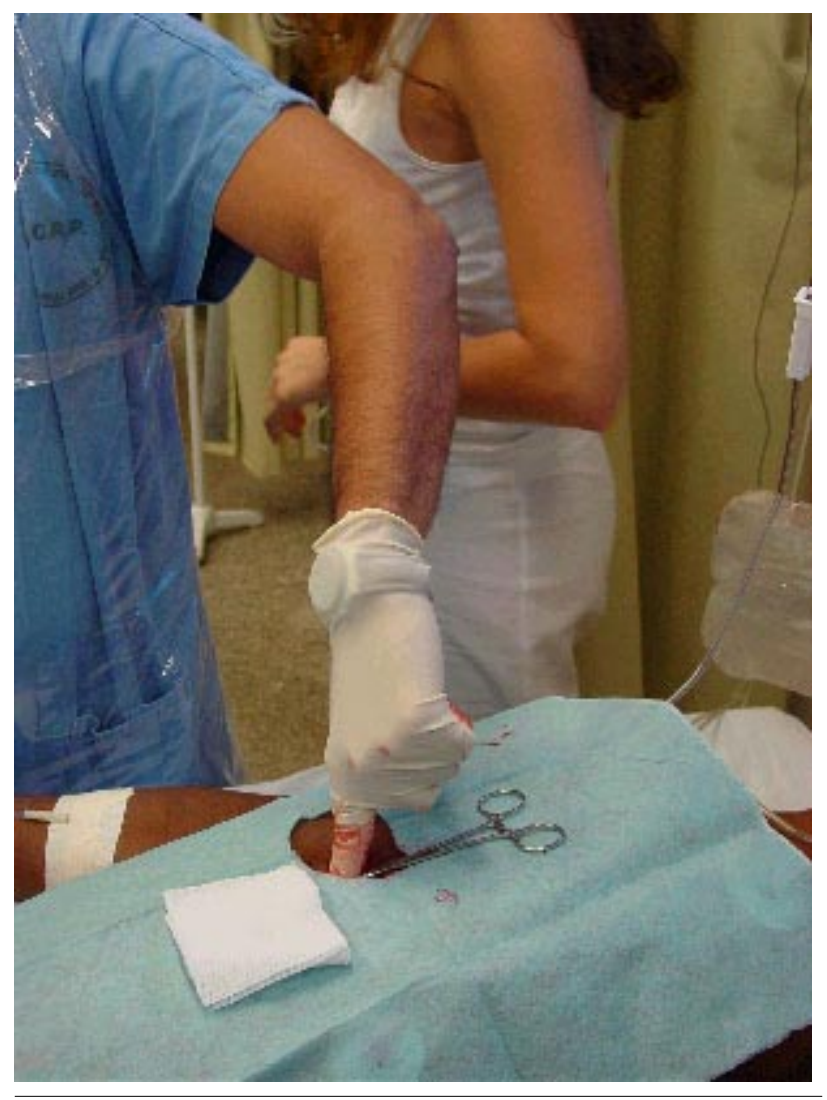

Figura 1. Exploração digital de ferimento por arma branca na parede abdominal anterior

O tratamento não operatório de pacientes com ferimentos por arma de fogo está ganhando aceitação num subgrupo de pacientes altamente selecionados que apresentam estabilidade hemodinâmica e sem sinais de peritonite ${ }^{15}$.

Embora o exame físico permaneça essencial na avaliação destes pacientes, outras técnicas diagnósticas, tais como a tomografia computadorizada, lavado peritoneal diagnóstico e a laparoscopia permitem acurada determinação de lesões intra-abdominais ${ }^{15}$.

A habilidade de excluir a presença de lesões de órgãos internos que necessitem de tratamento cirúrgico evita as complicações potenciais das laparotomias desnecessárias ${ }^{15}$.

\subsection{4- Ferimentos penetrantes da transição toracoabdominal}

Em pacientes assintomáticos, as opções diagnósticas são o exame físico seriado, radiografia simples seriada de tórax, toracoscopia, laparoscopia ou tomografia computadorizada (para ferimentos toracoabdominais do lado direito). Mesmo com todas estas opções diagnósticas, as hérnias diafragmáticas pós- 
traumáticas do lado esquerdo continuam a ocorrer em pacientes com ferimentos toracoabdominais por arma branca. Em casos de ferimentos toracoabdominais do lado esquerdo por arma de fogo, a conduta mais segura é a laparotomia. ${ }^{16,17}$

\section{6- EXAMES COMPLEMENTARES}

\section{1- Exames laboratoriais}

Além da tipagem sangüínea e das provas cruzadas, amostras de sangue podem ser utilizadas para medidas de hematócrito/hemoglobina, leucometria, amilasemia, a dosagem de álcool ou outras drogas e testes de gravidez nas mulheres traumatizadas em idade fértil ${ }^{1}$.

Os valores iniciais de hematócrito e hemoglobina não refletem a quantidade de sangramento intraabdominal. Necessitam-se várias horas para que ocorra hemodiluição e para que haja reflexo nos valores do hematócrito. Assim, seu valor inicial pode servir como base de comparação ${ }^{1}$.

Exames laboratoriais seriados, caracterizando mudanças como a queda da hematimetria, o aparecimento de leucocitose e o aumento da amilase podem ser indícios de lesão abdominal oculta.

O exame de urina pode, eventualmente, auxiliar na constatação da presença de micro-hematúria, sugerindo a presença de lesão no sistema urinário em pacientes que estejam hemodinamicamente instáveis na admissão.

\section{2- Exames radiológicos}

\subsection{1- Radiografias simples}

A radiografia simples de abdome é de pouca contribuição diagnóstica no trauma abdominal.

No trauma contuso de abdome, somente quando se deseja identificar a presença de ar livre na cavidade peritoneal (pneumoperitôneo - Figura 2) e a integridade das cúpulas diafragmáticas, a radiografia simples de torax com cúpulas poderá caracterizar uma provável rotura de víscera oca ou hérnia diafragmática. Se o paciente não puder ficar sentado ou de pé para a realização da radiografia devido à dor ou suspeita de fratura vertebral, a radiografia de abdome deve ser realizada com o paciente em decúbito lateral esquerdo $^{8}$.

A radiografia simples do abdome, em posição ortostática e supina, pode identificar a presença de ar retroperitoneal (retropneumoperitônio), geralmente, pela melhor definição dos rins ou da sombra do músculo psoas. A presença de apagamento da sombra do músculo psoas sugere lesão retroperitoneal com hematoma. Outros sinais como velamentos ou distensões gasosas são inespecíficos. No entanto, o achado de fraturas das costelas inferiores, da coluna lombar, dos processos transversos de vétebras lombares e da pelve pode sugerir a concomitância de lesões dos órgãos adjacentes, principalmente, baço, fígado e dos rins ${ }^{8}$

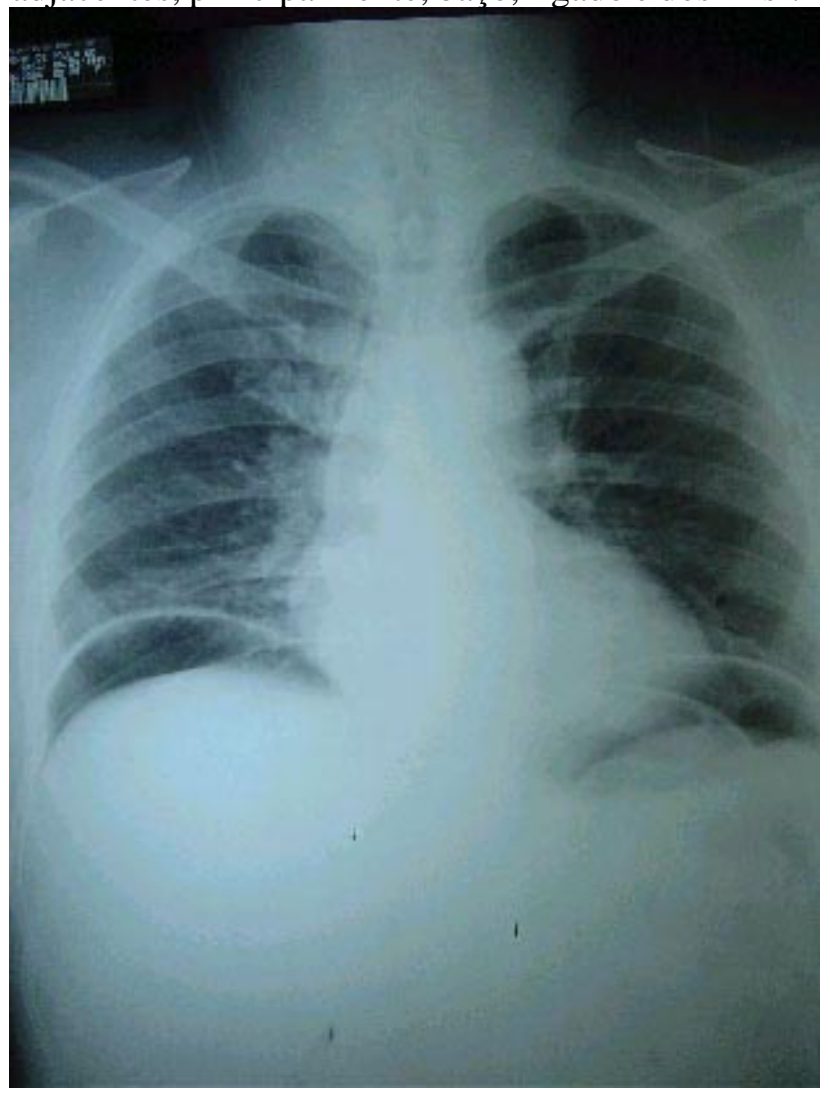

Figura 2. Presença de pneumoperitônio em radiografia simples de tórax com cúpulas.

Nos casos de trauma abdominal penetrante por arma de fogo, os pacientes que estão instáveis hemodinamicamente devem ser levados imediatamente para exploração cirúrgica. Se o estado hemodinâmico estiver normal, os orifícios de entrada e saída devem ser marcados com clips ou qualquer outro material radiopaco para se ter uma idéia da trajetória presumi$\mathrm{da}^{8}$ (Figura 3).

Outra utilidade da radiografia simples de abdome é para detectar a presença de corpos estranhos radiopacos.

A radiografia simples da pelve faz parte da investigação radiológica de rotina do paciente politrau- 


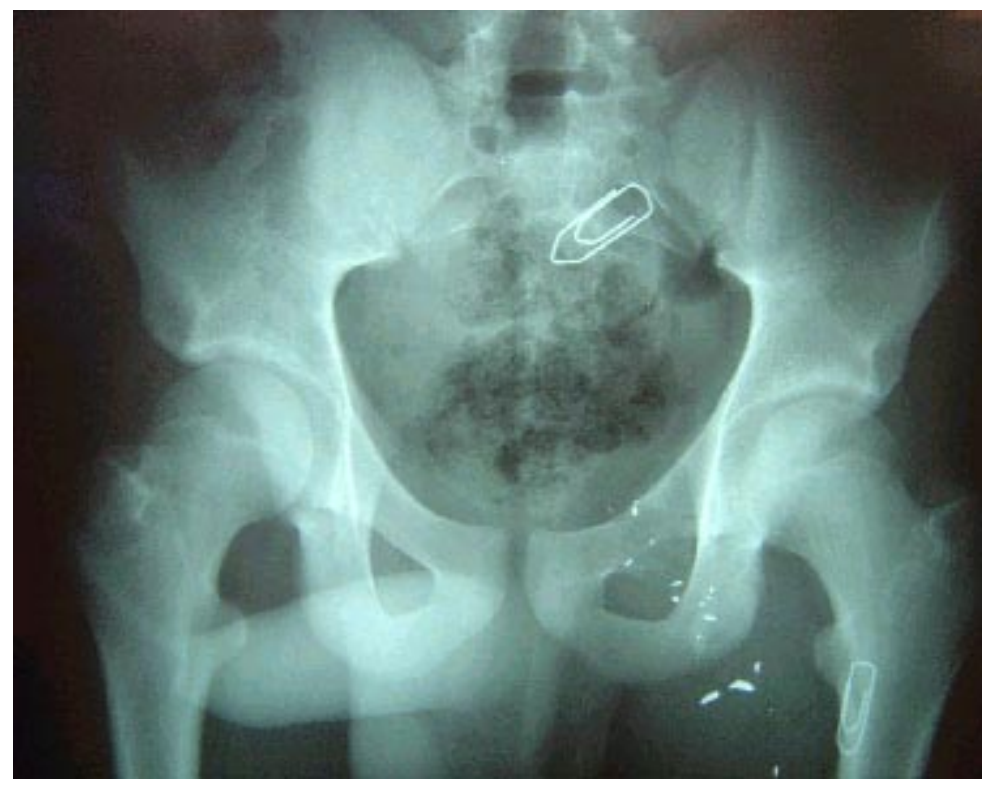

Figura 3. Presença de clips metálicos indicando os orifícios de entrada e saída do projétil de arma de fogo em radiografia simples de pelve para definir a trajetória.

matizado, particularmente naqueles pacientes cujo exame clínico é pouco confiável, como nos pacientes com escore de coma de Glasgow menor que 12 ou com falta de resposta ao estímulo doloroso. Nos demais pacientes, com exame clínico confiável, pode não ser necessária a sua utilização ${ }^{1}$.

\subsection{2- Radiografias contrastadas}

Os estudos radiológicos contrastados poderão ser empregados em pacientes que apresentam estabilidade hemodinâmica, cuja indicação traga uma real contribuição diagnóstica.

A uretrocistografia injetora (Figura 4) está indicada nos casos de suspeita de lesão uretral (uretrorragia, hematoma de períneo e próstata em posição elevada ou não palpável no toque retal), antes da introdução de sonda vesical ${ }^{8}$.

Em pacientes com sonda vesical e com suspeita de lesão de bexiga, a cistografia, conseguida pela repleção vesical com 250 a $300 \mathrm{ml}$ de contraste hidrossolúvel, seguida de três incidências (ântero-posterior, perfil e após esvaziamento vesical), é especialmente utilizada nos pacientes com traumas pélvicos apresentando hematúria macroscópica ${ }^{8}$.

A urografia excretora pode ser utilizada em casos com suspeita de trauma renal, porém a tomografia computadorizada permite melhor caracterização do tipo e extensão das lesões, bem como a presença de lesões associadas.
A seriografia de esôfago, estômago e duodeno e o enema baritado do cólon são menos freqüientemente realizados em casos particulares de suspeita de lesões de duodeno e cólon retroperitonial, que não apresentam manifestações clínicas precoces ${ }^{8}$.

\subsection{3- Ultrassonografia abdominal}

A maioria dos estudos de ultrassonografia abdominal em pacientes politraumatizados recomenda a sua utilização como exame diagnóstico inicial, teste de "screening" (Focused Assessment for Sonography in Trauma - FAST) ou estudo adjuvante complementar à tomografia computadorizada ou ao lavado peritoneal diagnóstico, devendo ser realizado na própria sala de admissão por médico capacitado ${ }^{18,19}$.

O objetivo do exame é a detecção e quantificação do hemoperitônio para identificar os pacientes com lesão e não o diagnóstico do órgão lesado. Sua sensibilidade está entre 80 e $99 \%$ na detecção de hemorragia intraabdominal $^{18,19}$.

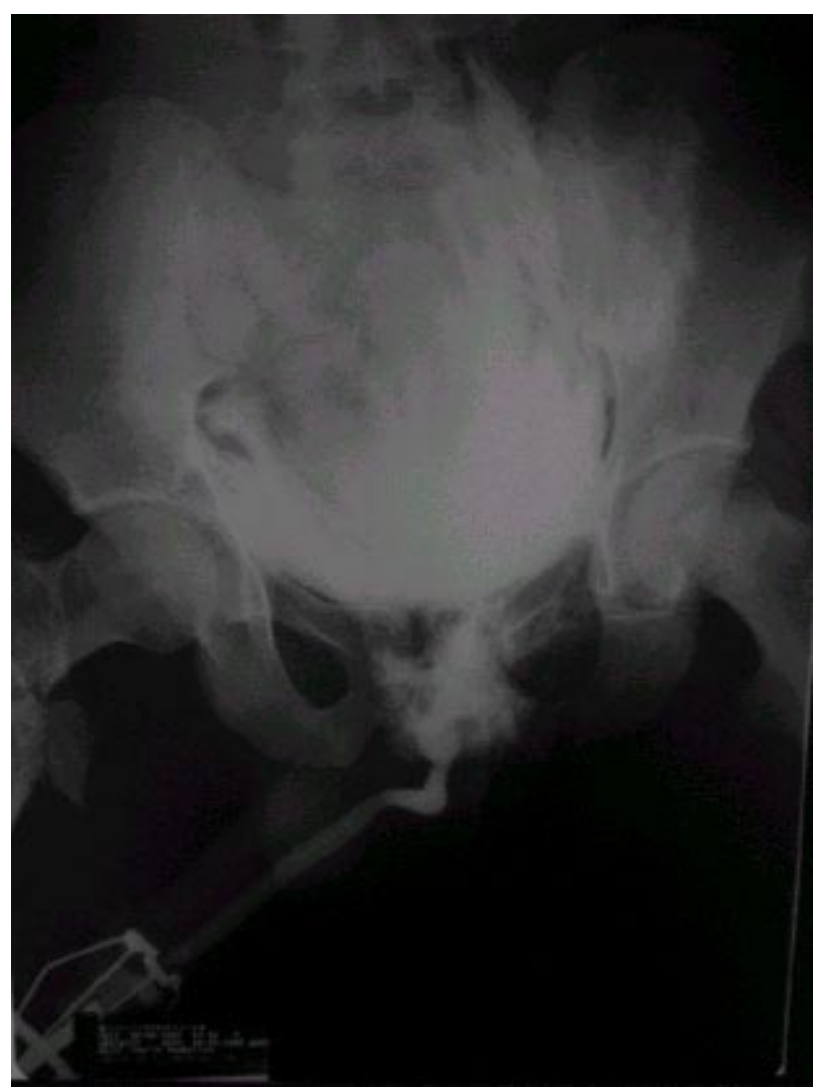

Figura 4. Uretrocistografia injetora mostrando lesão de uretra e ruptura intraperitoneal de bexiga. 
$\mathrm{Na}$ sistematização da ultrassonografia abdominal (FAST) para trauma são examinadas quatro regiões à procura de líquido livre: saco pericárdio, fossa hepatorrenal (Espaço de Morrison), fossa esplenorenal e a pelve. Após o exame inicial, o mesmo pode ser repetido para detectar hemoperitônio progressivo ${ }^{18,19}$.

A ultrassonografia tem substituído o lavado peritoneal diagnóstico em função das suas vantagens ${ }^{18}$ : é de fácil utilização, pode ser portátil, é de rápida execução, pode ser repetido, e não tem risco dos efeitos da radiação ${ }^{19}$ (Figura 5).

Alguns fatores comprometem a utilização da ultrassonografia abdominal tais como a obesidade, a presença de enfisema sub-

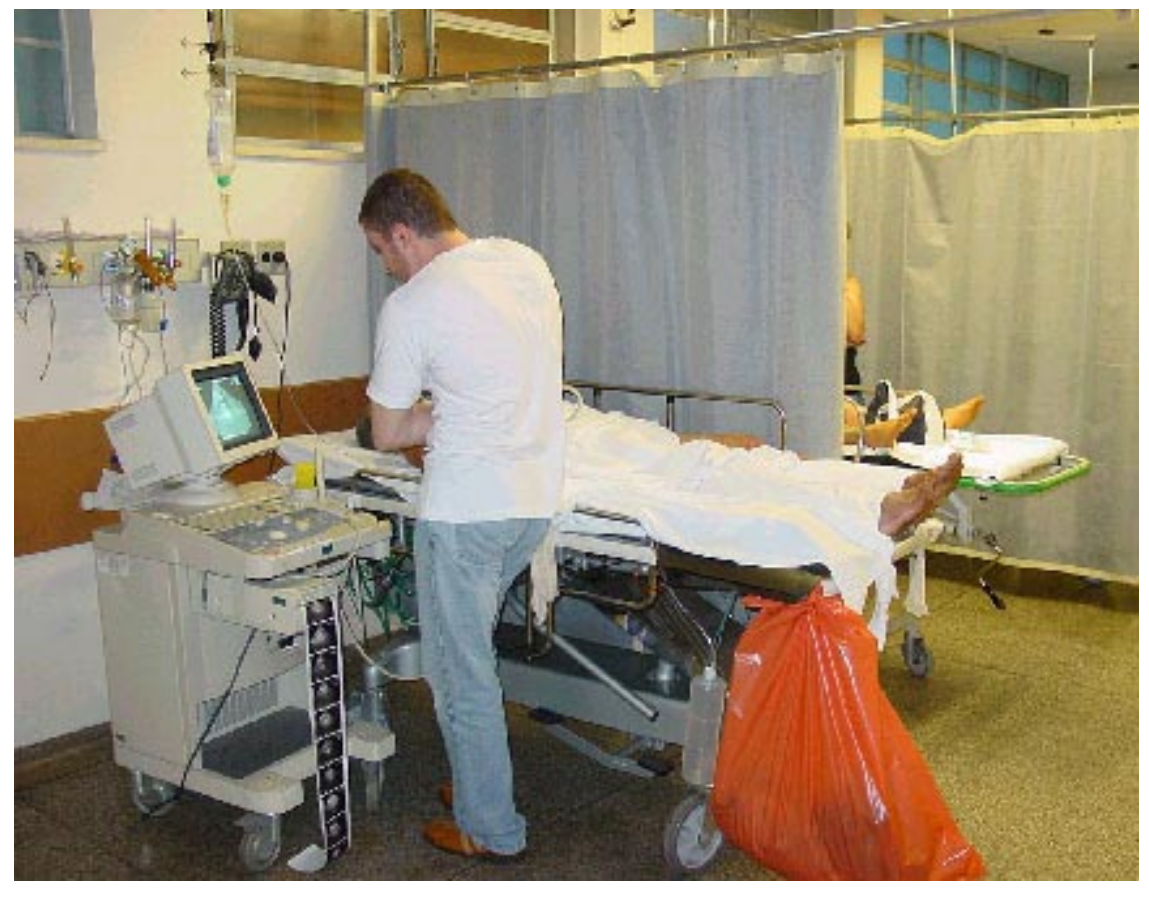

Figura 5. Exame de ultrassom (FAST) realizado à beira do leito na sala de trauma. cutâneo, presença de distensão abdominal por gases e cirurgias abdominais prévias ${ }^{19}$.

\subsection{4- Tomografia computadorizada}

A tomografia computadorizada não deve ser realizada em pacientes hemodinamicamente instáveis e naqueles com sinais óbvios de peritonite que requerem exploração cirúrgica imediata. Requer o transporte do paciente para o setor de radiologia e devem-se tomar todos os cuidados neste transporte e na monitorização do paciente durante a realização do exame ${ }^{8,20}$.

Contrastes hidrossolúveis administrados por via oral e venosa proporcionam melhores resultados na interpretação das imagens e, portanto, devem ser utilizados. O contraste oral permite avaliar o trato gastrintestinal superior e o uso endovenoso permite avaliar a integridade do órgão e avaliar o fluxo vascular. Os cortes tomográficos de 1 a 2 centímetros devem abranger o tórax inferior e toda a pelve. Em traumas penetrantes do dorso e flanco, a utilização adicional do contraste por enema, melhora a sensibilidade para lesões retroperitoneais do cólon ${ }^{8}$.

A tomografia computadorizada não apresenta uma boa sensibilidade e especificidade nas lesões gastrointestinais, diafragmáticas e pancreáticas. $\mathrm{Na}$ ausência de lesões hepática e esplênica, a presença de líquido livre na cavidade peritoneal sugere uma lesão do trato gastrointestinal e/ou de seu mesentério ${ }^{8,20}$.

Assim, os pacientes com trauma abdominal contuso que apresentam líquido livre isolado na tomografia computadorizada e sem lesões de vísceras parenquimatosas não precisam necessariamente serem submetidos à laparotomia exploradora. Os pacientes alertas podem ser seguidos com exames físicos repetidos e aqueles com alteração do nível de consciência devem ser submetidos ao lavado peritoneal diagnóstico ${ }^{21}$.

Na tentativa de padronizar o conhecimento para facilitar a pesquisa clínica, a "American Association for the Surgery of Trauma" propôs a classificação da Organ Injury Scaling (OIS). Tal classificação é utilizada para o estadiamento das lesões abdominais por meio da realização dos exames radiológicos ou pelo achado intra-operatório ${ }^{22}$.

A grande vantagem da tomografia computadorizada de abdome é permitir o estadiamento anatômico das lesões dos diferentes órgãos abdominais. As lesões de grau 1 a 3 são consideradas minor e as lesões de grau 4 e 5 são classificadas como major. Esta graduação das lesões auxilia o cirurgião na tomada de decisão em relação à indicação cirúrgica ${ }^{22}$.

\section{3- Lavado peritoneal diagnóstico (LPD)}

O lavado peritoneal é muito sensível para a detecção de hemorragia (98\%), é rápido e simples de ser realizado e não requer equipamento sofisticado ${ }^{1}$. Entretanto, o lavado peritoneal não diferencia entre 
sangramento de pequenas lesões daqueles significantes, resultando em laparotomias desnecessárias em 6 a $25 \%$ dos casos. Também não mostra a localização ou extensão das lesões, nem mostra lesões do retroperitônio e, assim, não tem sensibilidade para as lesões traumáticas do pâncreas, rins e porção retroperitoneal do duodeno. Em ferimentos por arma de fogo não é confiável, tendo altos valores de falso negativo"

Assim, o lavado peritoneal diagnóstico está indicado nos pacientes politraumatizados que apresen$\operatorname{tam}^{1,9}$ :

- exame físico abdominal de interpretação duvidosa, por apresentar dor abdominal que pode ser atribuída às fraturas de costelas inferiores, fratura pélvica ou lombar,

- exame físico abdominal não confiável, como nos pacientes com alteração da consciência devido ao trauma de crânio e/ou intoxicação por droga depressora do sistema nervoso central ou, por trauma raquimedular,

- impossibilidade de seguimento clínico do abdome em pacientes anestesiados para cirurgias em outros seguimentos corpóreos extra-abdominais ou submetidos a exames complementares prolongados.

Por se tratar de procedimento cirúrgico, não insento de risco, o LPD deve ser feito, preferencialmente, por um cirurgião. Como o método altera o exame físico subsequente do abdome, o cirurgião que irá tratar, receber e conduzir o doente, é o profissional mais indicado para realizar o procedimento. Tal procedimento não deve ser realizado num serviço de saúde sem possibilidade de oferecer o tratamento definitivo ao paciente traumatizado ${ }^{1}$.

A única contra-indicação absoluta do LPD é a laparotomia exploradora já indicada. As demais contra-indicações são todas relativas: obesidade mórbita, gravidez, cirurgias abdominais prévias, coagulopatia pré-existente e a cirrose avançada. Pode ser feito por técnica de Seldinger e pela técnica aberta, que é mais segura e rápida ${ }^{9}$. A técnica aberta é apresentada na Tabela I.

Os critérios de positividade do LPD são:

1- saída de mais de $10 \mathrm{ml}$ de sangue na aspiração inicial após abertura do peritônio;

2- saída de sangue à drenagem do líquido infundido;

3- mais de 100.000 hemácias por campo ou 500 leucócitos no exame do líquido de retorno; e

4- saída de restos alimentares, bile e material fecal. Quando se utiliza o LPD em ferimentos penetrantes por arma branca da região toracoabdominal, pelo risco de lesão diafragmática, considera-se positivo qualquer valor acima de 5.000 hemácias $/ \mathrm{mm}^{3}$.

$\mathrm{O}$ aumento de glóbulos brancos ocorre em resposta inflamatória do peritônio a material estranho, especialmente em ferimentos de vísceras ocas, porém é necessário um lapso de 3 a 5 horas para que este valor tenha utilidade, pois antes desse período a sua sensibilidade é baixa ${ }^{1,9}$.

Caso o exame seja negativo, retira-se o cateter e sutura-se a aponeurose e a pele. Caso o exame seja positivo, apenas retira-se o cateter, faz-se um curativo e encaminha o paciente ao centro cirúrgico.

Tabela I: Técnica do lavado peritoneal diagnóstico

1. Passagem de sonda gástrica e vesical.

2. Assepsia e antisepsia.

3. Anestesia local com xilocaína.

4. Incisão longitudinal de $2 \mathrm{~cm}$ inferior à cicatriz umbilical. Se houver suspeita de fratura de bacia, a incisão deve ser supraumbilical e, na gestante, a incisão deve ser realizada acima do fundo do útero.

5. Dissecção até o encontro da aponeurose. Hemostasia rigorosa.

6. Incisão da aponeurose longitudinalmente na linha média.

7. Dissecção com pinça hemostática - tipo Kelly até o encontro do peritônio. Hemostasia rigorosa.

8. Pinçamento do peritônio com Kelly. Realização de sutura em bolsa no peritônio.

9. Abertura do peritônio e introdução de cateter de diálise peritoneal na cavidade abdominal dirigido para baixo e para a esquerda.

10. Tentativa de aspiração de sangue com seringa conectada ao cateter. Se aspirar mais de $10 \mathrm{ml}$ de sangue, considera-se o exame positivo e está indicada a lapatoromia exploradora.

11. Se não houve a aspiração de sangue, infundir $1000 \mathrm{ml}$ de soro fisiológico aquecido no adulto ou $10 \mathrm{ml}$ por quilo na criança.

12. Drenagem do líquido infundido, por mecanismo de sifonagem, no próprio frasco de soro (Importante: não furar o frasco de soro a ser infundido com agulha).

13. Enviar material ao laboratório para análise (Quando necessário). 
Devemos lembrar que durante a realização do LPD, pequenas quantidades de ar penetram na cavidade peritoneal e isto pode ser interpretado como um resultado falso positivo de perfuração de víscera oca numa radiografia simples subseqüente. Por outro lado, a recuperação incompleta do líquido infundido pode resultar em falsa interpretação de hemoperitônio numa tomografia computadorizada ou ultrassom realizado posteriormente 9 .

\section{4- Laparoscopia}

Sua utilização no diagnóstico do trauma abdominal permanece limitada por várias razões:

1- necessidade de equipamento especial e pessoal treinado;

2- necessidade de sala cirúrgica e anestesia geral;

3- risco de embolia gasosa e pneumotórax hipertensivo pelo pneumoperitônio;

4- não pode ser realizada em pacientes instáveis; e

5- pelo alto custo ${ }^{17,23}$.

Algumas destas limitações estão diminuindo com a maior familiaridade com a técnica e com a experiência acumulada. Por exemplo: sua sensibilidade pode ser comparada à LPD e ao ultrassom, é segura e rápida, é específica e pode ser realizada em salas de emergência, com anestesia local, com pouco incômodo ao paciente ${ }^{23}$.

O papel da laparoscopia no trauma permanece controverso. Os estudos mais recentes mostram que a laparoscopia tem mais valor no trauma penetrante, evitando laparotomia em mais de dois terços dos pacientes com suspeita de lesão intra-abdominal. No trauma contuso, a laparoscopia pode servir como adjuvante da avaliação ${ }^{17,23}$.

É o melhor exame para o diagnóstico de lesões diafragmáticas, mas implica no risco de pneumotórax hipertensivo ${ }^{23}$.

\section{5- Toracoscopia}

Tem indicações específicas nos casos de trauma:

1- tratamento do hemotórax retido;

2- tratamento do pneumotórax persistente;

3- avaliação do diafragma nas lesões toraco-abdominais penetrantes;

4- tratamento de coleções infectadas do espaço pleural; e

5- diagnóstico e tratamento do sangramento contínuo em paciente hemodinamicamente estável ${ }^{24}$.

\section{7- TRATAMENTO}

\section{1- Tratamento não operatório}

O paciente deve permanecer em observação clínica e, dependendo do potencial para instabilidade hemodinâmica, deverá ficar em ambiente de terapia intensiva com monitorização contínua dos sinais vitais. Não deve receber analgésicos e nem antibióticos e estar em jejum por, pelo menos, 12 horas. Neste período, o paciente deve estar em repouso absoluto no leito, o exame físico deve ser repetido, de preferência, pelo mesmo observador e devem ser realizadas dosagens hematimétricas seriadas. Deve-se estar atentos ao desenvolvimento de hipotensão arterial, taquicardia, febre ou dor à palpação abdominal ${ }^{25}$.

\subsection{1- Trauma contuso}

O uso da tomografia computadorizada nos pacientes com trauma abdominal contuso propicia o tratamento não operatório das lesões de órgãos parenquimatosos, diminuindo a necessidade de cirurgia exploradora e reduzindo a freqüência de laparotomias não terapêuticas. A tendência atual em direção ao tratamento não operatório de muitas lesões do fígado, baço e rins é devido, em parte, à capacidade da tomografia computadorizada não apenas de definir a presença da lesão e a sua extensão, mas também de excluir outras lesões significantes, evitando cirurgias desnecessárias ${ }^{25,26}$.

A decisão final a respeito da indicação de exploração cirúrgica deve ser tomada com base nos achados tomográficos, em conjunção com o quadro clínico completo, principalmente, a estabilidade hemodinâmica e a necessidade de reposição de fluidos com soluções cristalóides e hemoderivados, além da avaliação pessoal de um cirurgião experiente ${ }^{25,26}$.

\subsection{2- Trauma penetrante}

Nos traumas abdominais penetrantes, apesar do forte consenso em favor da cirurgia mandatória, independentemente dos sinais clínicos apresentados, os ferimentos por arma branca começaram a ter uma abordagem mais seletiva, quando o paciente está relativamente assintomático (podendo referir dor no local do ferimento), permitindo a realização de exames físicos seriados, a exploração local do ferimento na parede abdominal anterior, o lavado peritoneal diagnóstico e a tomografia computadorizada com triplo contraste em ferimentos do flanco ou dorso ${ }^{12,14}$. 
O sucesso da orientação para tratamento não operatório para lesões de órgãos parenquimatosos em casos de trauma abdominal contuso e para casos selecionados de ferimentos por arma branca aceitos na maioria dos grandes centros de trauma, encorajaram a conduta conservadora não operatória em pacientes com ferimentos penetrantes por arma de fogo. Apesar do risco de lesão associada em mais de $95 \%$ dos casos, tal conduta passou a ser adotada no subgrupo de pacientes sem penetração da cavidade peritoneal, que algumas vezes pode ser clinicamente óbvia e, outras vezes, requer a confirmação através de exames de imagem, particularmente, a tomografia computadorizada $^{14,15,20}$.

Dois argumentos utilizados para justificar o tratamento não operatório para ferimentos penetrantes abdominais, tanto por arma branca, quanto por arma de fogo, tem sido a significante redução da morbidade, os custos associados à laparotomia não terapêutica e o potencial para cicatrização de órgãos parenquimatosos (fígado, baço e rins) traumatizados, como observado no tratamento de lesões graves desses órgãos no trauma contuso ${ }^{15,27}$.

O objetivo da adoção de uma conduta não operatória é minimizar a incidência de exploração negativa sem aumentar a morbidade de uma lesão desapercebida $^{28}$.

Nos casos de ferimentos por arma branca, atualmente, prevalece a conduta seletiva, visto que $50 \%$ das laparotomias são negativas, se a cirurgia for mandatória ${ }^{29}$.

Certos fatores devem ser considerados na seleção dos pacientes para tratamento não operatório, sendo que o primeiro deles é a estabilidade hemodinâmica ${ }^{29}$.

Um paciente com instabilidade hemodinâmica, que não responde à reposição de fluidos, requer exploração cirúrgica imediata. Num paciente com estabilidade hemodinâmica, definido como uma pressão sistólica maior que $90 \mathrm{mmHg}$, a avaliação clínica, laboratorial e o estadiamento radiológico deve ser realizado para quantificar adequadamente o grau de lesão e a presença de lesões associadas. O exame físico deve incluir a exploração local do ferimento, embora a avaliação da integridade fascial possa ser difícil e pouco acurada no paciente jovem e com musculatura abdominal desenvolvida ${ }^{12,13,28,29}$.

Alguns trabalhos mostram a presença de lesões orgânicas significantes em apenas 21,4\% dos ferimentos por arma branca dos flancos e $7 \%$ dos ferimentos por arma branca do dorso ${ }^{12,13,14}$.

A baixa incidência de lesões por ferimentos no dorso é atribuída à espessura da musculatura e as barreiras esqueléticas ${ }^{14}$.

A tomografia computadorizada com triplo contraste (via oral, endovenosa e por enema) deve ser utilizada como exame primário para traumas penetrantes do flanco e do dorso, em pacientes com estabilidade hemodinâmica e sem sinais óbvios de peritonismo $^{14,23,24}$

Um risco potencial do tratamento não operatório de ferimentos por arma branca é a lesão diafragmática desapercebida. Os achados radiológicos na tomografia computadorizada não são específicos. A mortalidade após herniação transdiafragmática decorrente de ferimentos por arma branca pode chegar a $36 \%$. A herniação ocorre através da lesão diafragmática esquerda, uma vez que à direita é tamponada pelo fígado ${ }^{23,24}$.

\section{2- Tratamento cirúrgico}

Os pacientes com sinais de irritação peritoneal, instabilidade hemodinâmica ou sangramento retal após ferimentos penetrantes, por arma branca ou arma de fogo do tronco devem ser encaminhados para exploração cirúrgica imediata ${ }^{29}$.

Os pacientes com instrumentos empalados no abdome devem ser submetidos à laparotomia exploradora para a retirada do mesmo sob visão direta (Figura 6).

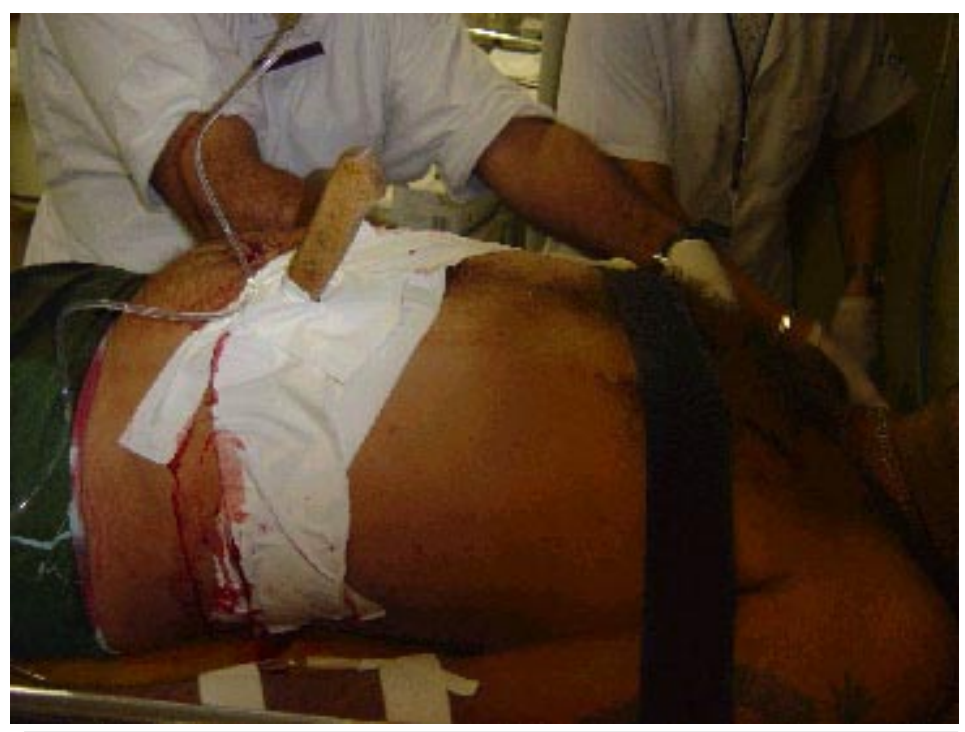

Figura 6. Paciente com arma branca empalada no abdome. 
Um conceito importante que precisa ser incorporado é a abordagem cirúrgica com "controle de danos" (Damage control) ou laparotomia abreviada. Trata-se da manutenção de um ambiente anatômico estável para impedir a progressão das alterações fisiológicas para um estado metabólico irreversível, uma vez que os pacientes morrem mais freqüentemente de déficits funcionais (hipotermia, acidose metabólica intratável e estado de incoagulabilidade sanguínea) do que do reparo anatômico completo dos órgãos ${ }^{30}$.

A opção por esta abordagem cirúrgica deve ser tomada tão logo se constate os déficits metabólicos do paciente e faça os controles dos sangramentos e das contaminações intestinais mais grosseiras. É feita a compressão da cavidade peritoneal, com os órgãos mantidos na sua posição anatômica utilizando-se compressas cirúrgicas (packing abdominal) e, é realizada a síntese temporária da parede abdominal. O paciente é internado em ambiente de terapia intensiva para controle dos déficits fisiológicos e, em 24 a 48 horas, após o controle da temperatura corpórea, da coagulação sangüínea e da acidose metabólica, será levado novamente ao centro cirúrgico para a correção anatômica definitiva das lesões ${ }^{30}$.

Outra abordagem cirúrgica que pode ser utilizada em situações especiais são as relaparotomias programadas (second look), principalmente, no caso de dúvidas sobre a viabilidade de alças intestinais, anastomoses com risco de deiscência, necrosectomias e infecções peritoneais graves ${ }^{30}$.

\section{3- Indicações de laparotomia exploradora}

Alguns achados clínicos e de exames complementares determinam a indicação operatória, como ${ }^{1}$ :

- Evidência de ferimento penetrante abdominal em paciente com instabilidade hemodinâmica.

- LPD francamente positivo em vítima de trauma fechado abdominal com hipotensão.

- Sinais evidentes de irritação peritoneal na admissão ou na evolução em pacientes com ferimentos penetrantes.

- Empalamento no abdome.

- Sangramento do estômago e reto em ferimentos penetrantes.

- Sinais de trauma abdominal com hipotensão recorrente apesar da reposição de fluidos.

- Pneumoperitôneo na radiografia simples de torax com cúpulas ou de abdome.

- Sinais radiológicos de lesão diafragmática.

- Lesão de alguma víscera oca revelada pela tomografia computadorizada.

- Evidência de lesão nos exames radiológicos contratados:

- extravasamento intraperitoneal em lesões de bexiga;

- extravasamento de contraste em lesões de uretra;

- extravasamento de contraste em lesões esofágicas, gástricas ou duodenais.

Pereira Júnior GA, Lovato WJ, Carvalho JB, Horta MFV. Management of the abdominal trauma. Medicina (Ribeirão Preto) 2007; 40 (4): 518-30, oct./dec.

ABSTRACTS: Abdominal trauma is regularly encountered in the emergency department. The lack of historical data and the presence of distracting injuries or altered mental status, from head injury or intoxication, can make these injuries difficult to diagnose and manage. Victims of trauma often have both abdominal and extraabdominal injuries, further complicating care.

This topic review will discuss a basic approach to the management of patients with blunt and penetrating abdominal trauma, including diagnostic tests and initial treatment considerations.

Keywords: Evaluation. Abdominal Injuries. Wounds, Penetrating. Wounds, Nonpenetrating. Treatment. 


\section{REFERÊNCIAS}

1 - American College of Surgeons. ACS. Comittee on Trauma. Advanced Trauma Life Support. Instructor manual. 7th ed, Chicago; 2004.

2 - National Association of Emergency Medical Technicians in Cooperations with the Comittee on Trauma of the American College of Surgeons. NAEMT. PHTLS basic and advanced prehospital trauma life support. 4th ed. Ohio; 1999.

3 - Shorr RM, Gottlieb MM, Webb K, Ishiguro LI, Berne TV. Selective management of abdominal stab wounds: importance of the physical examination. Arch Surg 1988; 123:1141-5.

4 - Feliciano DV, Burch JM, Spjut-Patrianely V, Mattox KL, Jordan GL Jr. Abdominal gunshot wounds. An urban trauma center's experience with 300 consecutive patients. Ann Surg 1988; 208:362-70.

5 - Schurink GW, Bode PJ, van Luijt PA, van Vugt AB. The value of physical examination in the diagnosis of patients with blunt abdominal trauma: a retrospective study. Injury 1997; 28(4): 261-5.

6 - Velmahos GC, Toutouzas KG, Radin R, Chan L, Demetriades D. Nonoperative Treatment of blunt injury to solid abdominal organs. Arch Surg 2003, 138: 844-51.

7 - Gilroy D, Saadia R, Hide G, Demetriades D. Penetrating injury to the gluteal region. J Trauma 1992; 32: 294-7.

8 - Mirvis SE, Hastings G, Scalea TM. Diagnostic imaging, angiography, and interventional radiology in the trauma patient. In: Mattox KL, Feliciano DV, Moore EE, eds. Trauma. 4th ed. New York: McGraw-Hill; 2000. Cap. 14, p. 261-310.

9 - Rodriguez A, DuPriest RW Jr, Shatney $\mathrm{CH}$. Recognition of intra-abdominal injury in blunt trauma victims: a prospective study comparing physical examination with peritoneal lavage. Am Surg 1982; 48:457-9.

10 - Ertekin C, Yanar H, Taviloglu K, Güloglu R, Alimoglu O. Unnecessary laparotomy by using physical examination and different diagnostic modalities for penetrating abdominal stab wounds. Emerg Med J 2005; 22(11):790-4.

11 - Oreskovich MR, Carrico J. Stab wounds of anterior abdomen. Analysis of a management plan using local wound exploration and quantitative peritoneal lavage. Ann Surg 1983; 195: 411-9.

12 - Sugrue M, Balogh Z, Lynch J, Bardsley J, Sisson G, Weigelt $J$. Guidelines for the management of haemodynamically stable patients with stab wounds to the anterior abdomen. ANZ J Surg 2007; 77(8):614-20.

13 - Coppa GF, Davalle M, Pachter HL, Hofstetter SR. Management of the wounds of back and flank. Surg Gynecol Obstet 1984; 159: 514-8.

14 - Chiu WC, Shanmuganathan K, Mirvis SE, Scalea TM. Determining the need for laparotomy in penetrating torso trauma: a prospective study using triple-contrast enhanced abdominopelvic computed tomography. J Trauma 2001, $51(5): 860-8$.
15 - Pryor JP, Reilly PM, Dabrowski GP, Grossman MD, Schwab CW. Nonoperative management of abdominal gunshot wounds. Ann Emerg Med 2004; 43 (3):344-53.

16 - Badhwar V, Mulder D. Thoracoscopy in the trauma patients: What is its role?. J Trauma 1996; 40: 1047A.

17 - Ahmed N, Whelan J, Brownlee J, CharinV, Chung R. The contribution of laparoscopy in evaluation of penetrating abdominal wounds. J Am Coll Surg 2005; 201(2):213-6.

18 - Rozycki GS, Ochsner MG, Schmidt JA, Frankel HL, Davis TP, Wang D, Champion HR. A prospective study of surgeonperformed ultrasound as the primary adjuvant modality for injured patient assessment. J Trauma 1995; 39:492-8.

19 - Rhea JT, Garza DH, Novelline RA. Controversies in the emergency radiology. CT versus ultrasound in the evaluation of blunt abdominal trauma. Emerg Radiol 2004; 10(6):28995.

20 - Stuhlfaut JW, Anderson SW, Soto JA. Blunt abdominal trauma: current imaging techniques and CT findings in the patients with solid organ, bowel, and mesenteric injury. Semin Ultrasound CT MR 2007; 28(2):115-29.

21 - Rodriguez C, Barone JE, Wilbanks TO, Rha CK, Miller K. Isolated free fluid on computed tomographic scan in blunt abdominal trauma: a systematic review of incidence and management. J Trauma 2002; 53(1):79-85.

22 - Moore EE, Shackford SR, Pachter HL, McAnich JW, Browner DC, Champion HR et al. Organ injury scaling: spleen, liver and kidney. J Trauma 1989; 29: 1664-6.

23 - Chelly MR, Major K, Spivak J, Hui T, Hiatt JR, Margulies DR. The value of laparoscopy in management of abdominal trauma. Am Surg 2003; 69(11): 957-60.

24 - Ahmed N, Jones D. Video-assisted thoracic surgery: state of the art in trauma care. Injury 2004; 35(5):479-89.

25 - Velmahos GC, Toutouzas KG, Radin R, Chan L, Demetriades D. Nonoperative treatment of blunt injury to solid abdominal organs. Arch Surg 2003, 138: 844-51.

26 - Sartorelli KH, Frumiento C, Rogers FB, Osler TM. Nonoperative management of hepatic, splenic, and renal injuries in adults with multiple injuries. J Trauma 2000; 49: 56-62.

27 - Saadia R, Degiannis E. Non-operative treatment of abdominal gunshot injuries. Br J Surg 2000; 87: 392-7.

28 - Ferrada R, Birolini D. New concepts in the management of patients with penetrating abdominal wounds. Surg Clin North Am 1999; 79:1331-56

29 - Demetriades D, Hadjizacharia P, Constantinou C, Brown C, Inaba K, Rhee P, Salim A. Selective nonoperative management of penetrating abdominal solid organ injuries. Ann Surg 2006, 244: $620-8$

30 - Loveland JA, Boffard KD. Damage control in the abdomen and beyond. Br J Surg 2004; 91(9):1095-1101.

Recebido em 29/06/2007

Aprovado em 31/01/2008 